OPEN ACCESS

Edited by:

Zisis Kozlakidis,

International Agency for Research on

Cancer (IARC), France

Reviewed by:

Devika Kir,

University of Miami Hospital,

United States

Xinyu Sheng,

Zhejiang Hospital, China

${ }^{*}$ Correspondence:

Joao A. C. Lima

jlima@jhmi.edu

Specialty section:

This article was submitted to Infectious Diseases - Surveillance,

Prevention and Treatment,

a section of the journal

Frontiers in Medicine

Received: 27 August 2021

Accepted: 04 October 2021

Published: 08 November 2021

Citation:

Varadarajan V, Shabani M, Ambale Venkatesh B and Lima JAC (2021)

Role of Imaging in Diagnosis and

Management of COVID-19: A

Multiorgan Multimodality Imaging

Review. Front. Med. 8:765975.

doi: 10.3389/fmed.2021.765975

\section{Role of Imaging in Diagnosis and Management of COVID-19: A Multiorgan Multimodality Imaging Review}

\author{
Vinithra Varadarajan, Mahsima Shabani, Bharath Ambale Venkatesh and Joao A. C. Lima* \\ Department of Cardiology, Johns Hopkins School of Medicine, Baltimore, MD, United States
}

In this pandemic of Coronavirus disease 2019 (COVID-19), a vast proportion of healthcare resources, including imaging tools, have been dedicated to the management of affected patients; yet, the frequent reports of unknown presentations and complications of disease over time have been changing the usual standard of care and resource allocation in health centers. As of now, we have witnessed multisystemic symptoms requiring the collaboration of different clinical teams in COVID-19 patients' care. Compared to previous viral pandemics, imaging modalities are now playing an essential role in the diagnosis and management of patients. This widespread utility of imaging modalities calls for a deeper understanding of potential radiologic findings in this disease and identifying the most compatible imaging protocol with safety precautions. Although initially used for respiratory tract evaluation, imaging modalities have also been used for cardiovascular, neurologic, and gastrointestinal evaluation of patients with COVID-19. In this narrative review article, we provide multimodality and multisystemic review of imaging techniques and features that can aid in the diagnosis and management of COVID-19 patients.

Keywords: biomarkers, imaging, management, diagnosis, COVID-19

\section{INTRODUCTION}

Coronavirus disease 2019 (COVID-19), caused by a novel enveloped single-stranded RNA virus, SARS-CoV-2, has emerged as a pandemic leading to a global public health crisis of the unprecedented magnitude of mortality and morbidity $(1,2)$. The viral surface spike proteins bind to the human angiotensin-converting enzyme 2 (ACE2) receptor, expressed in the alveolar cells, vascular endothelium, and enterocytes of the intestine $(3,4)$. According to WHO guidelines, COVID-19 patients were divided into mild, moderate, severe, and critical stages based on symptoms, clinical signs, laboratory results, and imaging (5). The clinical spectrum of COVID-19 ranges from asymptomatic carriers who can transmit the virus to mild clinical upper respiratory infection, which can progress to an acute respiratory distress syndrome (ARDS) in critically ill patients (6). The most common symptoms in hospitalized patients were fever, dry cough, shortness of breath, nausea/vomiting, and diarrhea. In addition, anosmia, dysgeusia, and gastrointestinal symptoms were also reported among hospitalized patients (7). About one-quarter of hospitalized patients require intensive care treatment, most commonly due to hypoxemic respiratory failure (8). More than half of the ICU patients require mechanical ventilation. The clinical characteristics 
and complications of COVID-19 are not limited to pulmonary symptoms but include myocardial, renal, liver, thromboembolic manifestations, and neurological events (7). It is believed that SARS-CoV-2 causes direct viral tissue damage to extrapulmonary organs by attaching to ACE2 receptors. In addition, endothelial damage, thrombo-inflammation, and immune response dysregulation also contributed to the extrapulmonary manifestations of the disease (9).

Early detection and management of the disease are challenging as clinical symptoms are non-specific, and disease progression is quite rapid (8). The first-line diagnostic test of COVID-19 is carried out by real-time reverse-transcriptase PCR (RT-PCR) test from a nasopharyngeal swab, which reportedly has high specificity but low sensitivity ranging from 80 to $90 \%$ due to insufficient viral load or failure of nucleic acid extraction $(10,11)$. Due to a high number of false-negative results and non-specific biomarkers, radiological imaging has become a crucial tool in the early detection and risk stratification of COVID-19 disease. Given the impact of systemic inflammation and procoagulant activity of the disease, multimodality imaging can also aid in phenotyping the organ injury and dysfunction due to COVID-19. Thus, comprehensive insight into the imaging hallmarks of COVID19 is mandatory for early diagnosis, stratifying the disease severity, effective treatment, and identification of potential sequela of this infection. On the other hand, comprehensive multimodality imaging cannot be employed due to the strict safety precautions required in the management of these patients, such as patient transfer and infection control measures. Hence, this narrative review sets out to explore the role and potential of multimodality imaging techniques in COVID-19 and outline the key findings in each modality. We searched Embase, PubMed, Cochrane library, and Web of Science databases through July 2021. No language restrictions were applied. Keywords of "lung imaging in COVID-19," "brain imaging in COVID-19," cardiovascular imaging in COVID-19, "lung injury in COVID-19," "RV dysfunction in COVID-19," "abdominal imaging in COVID-19," "acute and chronic effects of COVID-19," "diagnosis and management of COVID-19" were utilized. We manually searched the reference lists of included articles and relevant reviews. We present the following article in accordance with a focus on diagnostic imaging implementation in North America.

\section{ROLE OF BIOMARKERS TO INFORM IMAGING DECISION MAKING}

Circulating biomarkers play a key role in the clinical judgment as it reflects organ injury, inflammation, dysfunction, and organ damage, which informs the management of the disease. Biomarkers have also been found to be the predictors of longer hospital stay, need for ICU admission, the onset of ARDS, and mortality $(12,13)$. In the acute stage of disease, markers of inflammation such as C-reactive protein (CRP), ferritin, lactate dehydrogenase, and coagulopathy such as prothrombin times, $\mathrm{D}$ dimer were reported to be elevated in most patients in addition to thrombocytopenia and lymphopenia (7). Cardiac biomarkers of myocardial injury (troponin) and myocardial stress (NTproBNP) have been reported in patients with myocardial stress/injury related to COVID-19 and are not specific to acute heart failure or myocardial infarction (12). Similarly, even though the elevation of liver function tests may be non-specific, it is commonly seen in other viral pneumonia and may indicate a liver injury and dysfunction (7). As the involvement of extrapulmonary organs is a major adverse prognostic finding in COVID-19, the links between circulating biomarkers and organ dysfunction make it tempting to regard them as a valuable tool for decision making regarding the need for imaging. It is appropriate to interpret that the need for imaging can be ignored if the biomarkers are mildly elevated and can be utilized in marked elevation as in severe COVID-19.

\section{ROLE OF IMAGING IN DIAGNOSIS}

\section{Initial Diagnostic Approach Chest Radiography}

Even though chest radiography is of little diagnostic value in the early stages due to high false-negative results, it has remained a technique often used for screening and disease severity assessment modality $(11,14)$. Equipment portability with imaging feasibility in the patient's room has made radiography favorable. A portable anteroposterior radiograph provides adequate information on lung findings associated with COVID-19, in addition to the assessment of lines and tubes and potential complications, such as pneumothorax, and maybe the only possible modality in severe infections.

At baseline, radiograph findings mostly include consolidation followed by hazy opacification $(10,11,15)$. The opacification was mostly seen in peripheral regions close to the pleura and lower lung zones with unilateral or bilateral involvement of the lungs $(11,16)$. Reports showed that as the disease progressed, the opacities increased in size and density, with multilobar involvement gradually leading to diffuse opacity, consolidation, and thickened interlobular septa as seen in the setting of ARDS (Figure 1) (10). Chest radiograph findings in COVID-19 infection may overlap with other viral pneumonia and, as a result, have lower sensitivity (69\%) than initial RT-PCR testing (11).

\section{Chest Ultrasound}

Lung ultrasound is easily accessible and widely used to diagnose, monitor, and manage patients in intensive care and emergency settings. Lung ultrasound has a higher sensitivity (94\%) and specificity (85\%) with a positive predictive value of $87 \%$ and a negative predictive value of $93 \%$ in diagnosing viral pneumonia, which leaves ultrasound as a potential assessment tool in COVID-19 infection $(17,18)$. Typical ultrasound findings in COVID-19 included pleural thickening, subpleural consolidation, B lines (multifocal or confluent), non-lobar and trans-lobar consolidation with air bronchograms, and small localized pleural effusions $(17,19)$. In the earlier symptomatic phase, pleural thickening with unilateral or bilateral B lines and spared areas were observed (19). In later stages, subpleural consolidations and visible air bronchograms were more dominant. In the recovery phase, there has been a regression of prior findings along with the re-emergence of A-lines $(17,19)$. 


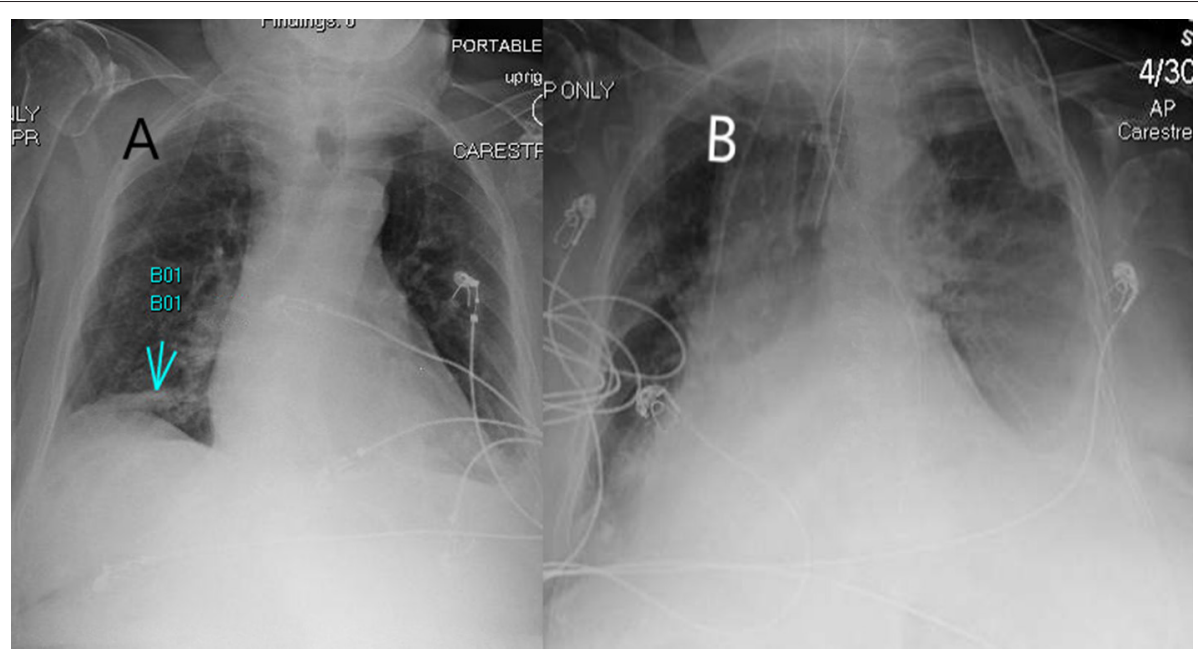

FIGURE 1 | A 82-year-old woman presented to the emergency department with cough, shortness of breath, altered mental status. (A) X-ray showing a right lower lobe infiltrate (arrow) on day 1 and tested positive for COVID-19 using PCR. Later, due to worsening respiratory function and confusion, the patient was admitted to the intensive care unit and was intubated. (B) Image showing small left pleural effusion with worsening infiltrates.

Low cost, ease of use, higher accuracy compared to chest X-ray (CXR), no risk of radiation exposure, and repeatability made lung ultrasound widely adopted in hospitals. The American Society of Echocardiography (ASE) proposed an algorithm and list of potential findings for the utility of Point-Of-Care UltraSound (POCUS) in the cardiac, lung, and vascular examination of patients with suspected or confirmed COVID-19 (Figure 2). The real-time feature, flexibility, and multiorgan application of POCUS have made it a proper initial diagnostic modality and an assessment tool for the hemodynamic status of critically ill patients (20). Furthermore, wireless ultrasound devices are now substituting physician stethoscopes for lung auscultation, regarding probe-mediated patient contact and ease of disinfection (21).

\section{Computed Tomography}

Although chest radiograph resembles CT findings, CT is more sensitive in detecting the abnormalities with a sensitivity of $98 \%$ $(11,22)$. Typical chest CT findings in patients with COVID19 include ground-glass opacity (GGO) (98\%), consolidation (64\%), reticular and crazy-paving pattern (36\%), predominantly in a bilateral, peripheral, posterior, and multilobar pattern (10, 11, 23-25). In earlier stages, GGO, hazy regions with increased density, and absence of broncho-vascular margins with a subpleural involvement of lower lobes either unilaterally or bilaterally have been reported to be the main CT findings (26, 27). Multifocal, patchy, or segmental consolidations have been considered as indicators of disease progression to the severe stage $(25,27,28)$. In the peak of the disease, GGOs with a crazypaving pattern, a thickened interlobular septa, and lines resulting from alveolar edema and interstitial inflammatory of lung injury were observed $(10,15,27,28)$. During the course of the disease and with aggravating symptoms, denser and diffusely distributed consolidation with more reticular configuration and thickened

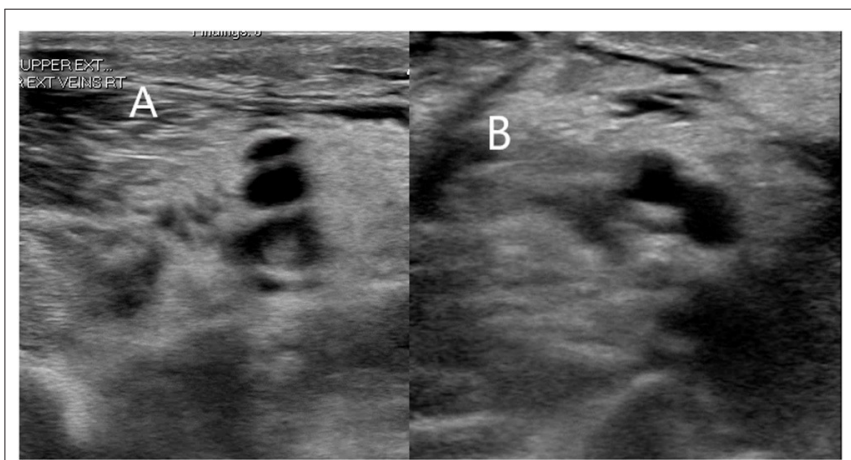

FIGURE 2 | A 70-year-old man in the intensive care unit had right arm swelling after 1 week of diagnosis of COVID-19. Doppler ultrasound of the right arm showing occlusive thrombus within right brachial (A) and basilic veins (B).

pulmonary interstitium due to lymphocyte infiltration were hallmarks $(10,26,28)$. There were resolutions of consolidations and crazy-paving patterns in the healing stage with extensive GGO (Figure 3) $(26,27)$. Except for a peripheral distribution and involvement of upper and middle lobes in COVID-19, imaging findings overlap with other non-COVID 19 pneumonia and are not specific (29). Currently, the American College of Radiology (ACR) does not recommend CT as a first-line diagnostic test for COVID-19 patients and limits CT utility to symptomatic and hospitalized patients with specific clinical indication, conditional to appropriate infection control procedures. However, decision making based on chest CTs is still an option only in facilities with a lack of access to sufficient testing (30).

\section{Advanced Imaging Techniques Magnetic Resonance Imaging}

Due to strict infection control measures, pulmonary MRI of COVID-19 is quite challenging and has not been well 


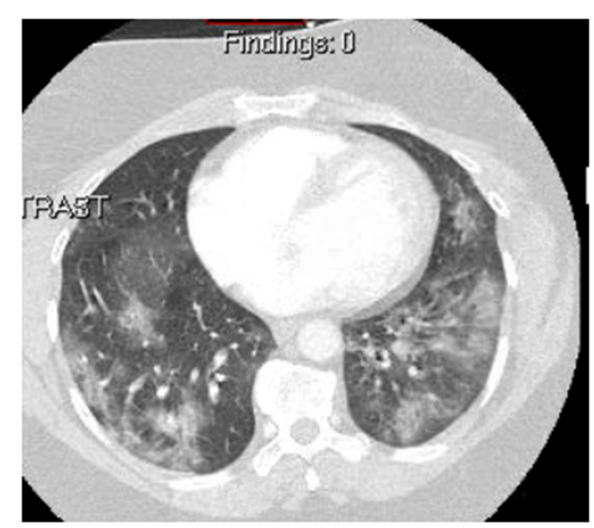

FIGURE 3 | A chest CT of a 35-year-old woman with no significant past medical history presenting with progressive dyspnea, chest pain, and hemoptysis showing typical ground-glass opacities and consolidation, which is seen in COVID-19. The patient was confirmed of COVID-19 with PCR testing.

TABLE 1 | Pulmonary imaging features of COVID-19.

\begin{tabular}{ll}
\hline Modality & Findings \\
\hline Radiograph & $\begin{array}{l}\text { Acute: Basal consolidation with hazy } \\
\text { opacification; unilateral/bilateral. } \\
\text { Progression: Diffuse and irregular opacification as seen } \\
\text { in ARDS. }\end{array}$ \\
& Acute: Ground-glass opacities (GGOs) unilateral or bilateral. \\
& Progression: Multifocal, patchy, or segmental consolidation. \\
& GGO with crazy-paving pattern. \\
& Severe: Reticular configuration, diffuse consolidation. \\
& Recovery: Progressive resolution. Disappearance of signs. \\
& Acute: Pleural thickening, subpleural consolidation, multifocal \\
BSG & P lines. \\
& Progression: Subpleural consolidation with visible \\
& Recovery: Re-emergence of A-lines. \\
& Acute: Cloudy sky sign with abnormal intensity using T1 and \\
& T2 sequences corresponding to GGO on CT. \\
Acute: Increased uptake corresponding to GGO on CT. & Increased nodal uptake. \\
MRI &
\end{tabular}

established except for a few case reports. MRI performed for non-pulmonary indications demonstrated incidental pulmonary findings consistent with CT (10). Pulmonary parenchymal focal zones can be visualized with abnormal intensity signals using T1 and T2 sequence as "cloudy sky sign" in acute settings, which can be correlated with GGO in CT (31).

\section{Fluorine-18-Fluorodeoxyglucose Positron Emission Tomography/CT $\left({ }^{18} \mathrm{~F}-\mathrm{FDG}\right.$-PET/CT)}

Another imaging modality, ${ }^{18} \mathrm{~F}-\mathrm{FDG} \mathrm{PET} / \mathrm{CT}$ is a non-invasive imaging method that identifies sites of inflammation and infection based on their higher metabolic demands (10). Few case studies reported increased uptake of ${ }^{18}$ F-FDG with a standardized uptake value of 4.6-12.2 in patients with peripheral GGO and lung consolidations detected by chest CTs $(32,33)$.
TABLE 2 | Cardiovascular imaging features of COVID-19.

\begin{tabular}{lll}
\hline Modality & Acute findings & Progressive findings \\
\hline Echocardiography & Increase in ejection function. & $\begin{array}{l}\text { RV dilation with or without } \\
\text { dysfunction followed by LV } \\
\text { dysfunction. Worsening RV } \\
\text { longitudinal strain. LV apical } \\
\text { ballooning with hyperkinetic }\end{array}$ \\
& & basal segments. \\
CT & & \\
& Reveals a filling defect due & \\
to pulmonary embolus, & \\
thrombus in the aorta. Can & \\
be used to assess plaque & \\
destabilization & \\
and progression. & \\
Increased wall thickness, & Diffuse interstitial myocardial \\
severe LV dysfunction, & edema by increased native \\
diffuse biventricular & T1 and ECV. LGE showed a \\
hypokinesis of apical & diffuse pattern suggestive of \\
segments. & acute myocarditis.
\end{tabular}

Notably, ${ }^{18}$ F-FDG PET/CT revealed an increased nodal uptake despite the absence of lymphadenopathy in COVID-19 (32, 33). Although ${ }^{18}$ F-FDG PET/CT cannot be used in emergency settings, it can play a complementary diagnostic role in COVID19 , especially when the symptoms are non-specific and diagnosis is challenging. Table $\mathbf{1}$ summarizes the pulmonary features of COVID-19.

\section{ROLE OF IMAGING IN MANAGEMENT}

Even though a typical presentation of COVID-19 was pulmonary symptoms, clinicians were frequently consulted to rule out cardiovascular, cerebrovascular, thrombogenic, and abdominal manifestations. Based on history, physical examination, biomarkers, ECG, and imaging modalities, clinicians can make an informed decision on the management.

\section{CARDIOVASCULAR IMAGING}

The cardiovascular complications of COVID-19, including myocardial infarction, myocarditis, acute-onset heart failure, thromboembolism, and cardiac arrest, are of great concern due to the higher case fatality rate observed in this subgroup of patients $(34,35)$. Myocardial involvement was associated with poor prognosis and more complicated adverse events, and therefore, there is a need for guidance on significant cardiac findings associated with COVID-19 (35-38). Table 2 summarizes the cardiovascular imaging findings of COVID-19.

\section{Initial Diagnostic Approach Echocardiogram}

In most COVID-19 patients, there have been signs of a hyperdynamic cardiac function, assumed to be due to systemic inflammation $(39,40)$. There have also been several reports of stress-induced (Takotsubo) cardiomyopathy observed as left ventricle (LV) apical ballooning with hyperkinetic basal segments 
$(39,41,42)$. Other frequently observed echocardiographic signs were right ventricle (RV) dilation (the $\mathrm{D}$-sign) with or without dysfunction, which is a poor prognostic factor and not just a sign of pulmonary embolism (PE), followed by LV diastolic and systolic dysfunction, pericardial effusion, and mild valvular heart disease (43-45). The majority of severe COVID-19 patients were found to have RV dilation/dysfunction along with elevated D-dimer, CRP, troponins, and cytokines (46). RV longitudinal strain was also found to provide prognostic information on COVID-19 $(46,47)$. In critically ill patients, hypoxic respiratory failure-induced pulmonary circulation injury causes severe RV dysfunction, which has a poorer prognosis and increased mortality in COVID-19 $(46,48)$. Consistently, severe global LV dysfunction and wall motion abnormalities have been observed in complicated cases of COVID-19 $(36,39)$.

Given the hypercoagulability induced by this virus, a phenomenon of pulmonary thrombosis in situ due to pulmonary involvement and local inflammation of the lung, rather than the migration of peripheral embolus, has been noted, which may suggest a different pathophysiologic mechanism of $\mathrm{PE}$ in this disease compared to $\mathrm{PE}$ described in other circumstances (49-51). Although echocardiography is not a sensitive tool in the early detection of pulmonary embolism, the secondary RV dysfunction signs, including severe free wall hypokinesia or akinesia with apical sparing (McConnell sign), shorter pulmonary acceleration time, and paradoxical interventricular septal movement, were prognostic markers for PE $(10,52,53)$.

Echocardiography is a safe, non-invasive technique that allows evaluation and quantification of global and regional cardiac function. The ASE guideline has recommended that POCUS, FoCUS (Focused Cardiac Ultrasound Study), UAPE (ultrasound-assisted physical examination), and bedside critical care echocardiography are preferred diagnostic modalities for $\mathrm{CV}$ manifestations of COVID patients (54).

\section{Advanced Imaging Techniques Magnetic Resonance Imaging}

Even though cardiac magnetic resonance imaging (CMR) is a safe, non-invasive, and preferred imaging modality in the quantification and characterization of myocardial tissue, the risk of contamination and imaging feasibility makes it challenging in acute settings. Increased wall thickness, diffuse biventricular hypokinesis of apical segments, severe LV dysfunction, and circumferential pericardial effusion was seen in the acute phases of infection (36). Moreover, the increased signal intensity of LV myocardium in T2-weighted images (T2WI) or native $\mathrm{T} 1$ and the increased extracellular volume (ECV) was suggestive of interstitial myocardial edema to identify the presence of myocardial inflammation in these patients (55). Late gadolinium enhancement (LGE) imaging showed diffuse-pattern involvement with predominantly subepicardial distribution, extended to the entire biventricular wall, fulfilling all Lake Louise criteria for the diagnosis of acute myocarditis $(36,55,56)$. Dedicated protocols with rapid acquisition to assess ventricular morphology and function (cine-images) and to detect myocardial edema (T2WI) in acute settings are encouraged (57).
On the other hand, decreased RV ejection fraction, cardiac output, stroke volume suggest RV myocardial edema, fibrosis, and impaired contractile function in patients recovered from COVID-19 (46).

\section{Computed Tomography}

Computed tomography angiography (CTA) is a rapid, noninvasive, broadly accessible, and highly accurate imaging method that can be utilized in hospitalized patients with elevated cardiac biomarkers. CTA has been replacing coronary angiography in several clinical settings and is an effective goalkeeper for invasive procedures, lowering costs, radiation, and adverse effects without compromising the patient outcome. CTA has also aided in visualizing the filling defects due to pulmonary embolus, or thrombus in the aorta, and has been preferred over $(16,58$, 59). Additionally, in accordance with guidelines, CT pulmonary angiography should be performed to rule out pulmonary embolism if supplementary oxygen was needed in COVID-19 patients with limited disease extension or when unenhanced CT findings could not explain the severity of the respiratory failure (60). Late contrast-enhanced cardiac CT has also been recommended as an alternative modality using dual-energy CT for myocarditis diagnostic when CMR use is not feasible, especially in patients with a planned concurrent lung CT (61). Findings in favor of myocarditis in cardiac CT are myocardial edema defined by a subepicardial late iodine enhancement, and a sub-endocardial perfusion defect, as observed in COVID-19 cases (62).

\section{CEREBROVASCULAR IMAGING}

The neurological manifestation of COVID-19 has been pleiotropic ranging from dizziness, anosmia, and dysgeusia in initial stages to immune-mediated vasculitis, stroke, and cranial neuropathies, and in full-blown disease, encephalomyelitis (63). In case of neurological emergencies like an acute stroke in COVID-19 patients, individual CT devices capable of angiography (CTA) and perfusion, multimodal MRI, and endovascular intervention units would be required to provide

TABLE 3 | Neurovascular imaging features of COVID-19.

\begin{tabular}{lll}
\hline Modality & Acute findings & Progressive findings \\
\hline MRI & $\begin{array}{l}\text { FLAIR images with hyperintensity } \\
\text { of the cortex and bilateral } \\
\text { olfactory bulbs with normal } \\
\text { volume. }\end{array}$ & $\begin{array}{l}\text { Infarct of cerebral arteries with } \\
\text { MRA revealing cerebral artery } \\
\text { stenosis. Diffuse hyperintensities } \\
\text { of bilateral temporal lobe and } \\
\text { thalamus. }\end{array}$ \\
& & $\begin{array}{l}\text { Rim enhancement on T1 } \\
\text { suggesting acute necrotizing } \\
\end{array}$ \\
& $\begin{array}{l}\text { Extensive hemorrhage with } \\
\text { CT }\end{array}$ & $\begin{array}{l}\text { Hypoattenuation localized to } \\
\text { bilateral medial thalami. }\end{array}$ \\
interventricular hypodensity. & Cerebral vein thrombosis. \\
Acute/subacute infarcts can & \\
be seen. &
\end{tabular}


proper care in a rapid time while not interfering with nonCOVID patients with the same acute presentations. Given this need, multiple stroke management algorithms have been designed and tested in multiple centers (64-66). Table 3 summarizes the imaging findings of the brain in COVID-19.

\section{Initial Diagnostic Approach Computed Tomography}

In the acute stages of COVID-19, brain CT showed no abnormalities, but later in the course of the disease, extensive acute subarachnoid and intraventricular hemorrhages along with cerebral swelling and hypodensity were reported in some patients $(63,67)$. In other studies, localized hypoattenuation in bilateral medial thalami causing acute necrotizing encephalopathy and cerebral vein thrombosis was visualized in brain CTs $(63,67)$. In patients with preexisting cardiovascular risk factors, there is a higher prevalence of acute ischemic strokes (Figure 4) (68).

In some instances, COVID-19 patients presented with varying degrees of peripheral neuropathy, especially Guillain-Barre syndrome (GBS). Neuroimaging has been recommended to be deferred in the case of neuromuscular disorders unless COVIDspecific precautions were not required (69). In GBS, spinal and brain MRI showed the enhancement of caudal nerve root and bilateral facial nerve, respectively $(63,70)$.

\section{Magnetic Resonance Imaging}

In the earlier stages of the disease in a patient with anosmia, brain MRI fluid-attenuated inversion recovery (FLAIR) showed cortical and bilateral olfactory bulb hyperintensity with normal sizes of olfactory bulbs $(63,71)$. As the disease progressed, the inflammatory and hypercoagulable state resulted in clinical strokes with brain MRI showing acute infarct of cerebral arteries and magnetic resonance angiogram (MRA) revealing stenosis of cerebral arteries $(63,67,72)$. In severe stages of the disease, brain MRI findings included diffuse hyperintensities of bilateral temporal lobe and thalamus, as well as nonconfluent multifocal white matter hyperintense lesions on FLAIR and extensive isolated white matter microhemorrhages. There have also been reports of internal hemorrhages indicated by hypointense signals on susceptibility-weighted imaging and rim enhancement on T1-weighted post-contrast imaging suggestive of acute necrotizing encephalopathy $(63,67,73-75)$.

\section{ABDOMINOPELVIC IMAGING}

Although a minority of patients have presented with digestiveonly manifestations, almost half of the confirmed cases of COVID-19 had both respiratory and digestive symptoms, with increased severity of the disease in those with gastrointestinalspecific manifestations, which mandates further evaluation by imaging (76). Table 4 summarizes the abdominopelvic imaging features of COVID-19.

\section{Initial Diagnostic Approach Abdominopelvic Radiography}

There were reports of portal venous gas suggestive of bowel infarction and signs of perforation in some cases (77).

\section{Ultrasound}

Additionally, ultrasound findings also revealed gallbladder distension, wall thickening, and sludge reflecting acute cholestasis (Figure 5), as well as increased echogenicity of hepatic parenchyma obscuring periportal echogenicity as markers of fatty liver, and mild pancreatitis revealed as the voluminous pancreas (77-79).

\section{Advanced Imaging Techniques Computed Tomography}

Computed tomography-derived signs in patients with COVID19 ranged from the fluid-filled colon in the early stages of diseases to more severe cases of local enterocolitis or even pancolitis with bowel wall thickening, small bowel distension, segmental bowel ischemia, pericolic fat stranding, pneumatosis intestinalis, mural hyperenhancement, and mesenteric hypervascularity ( 77,

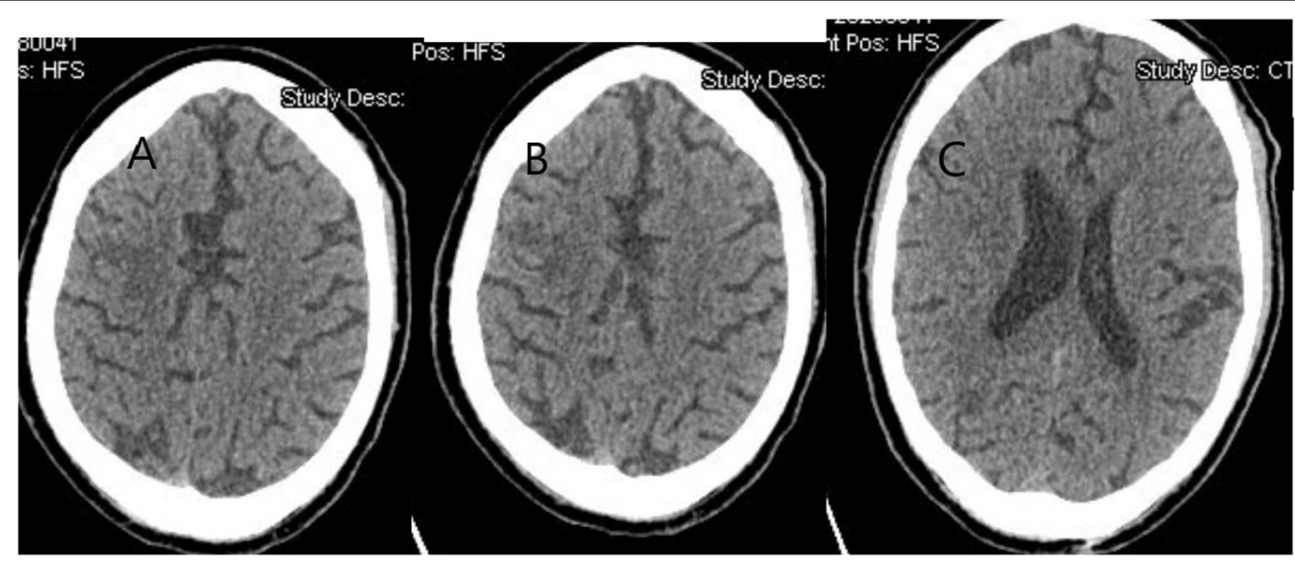

FIGURE 4 | A 68-year-old man complained of acute weakness of the left side of her body 3 days after being diagnosed with COVID-19. Brain CT without contrast shows multiple foci of infarcts in the right middle cerebral artery involving the right frontal, parietal, and temporo-occipital junction. 
TABLE 4 | Abdominopelvic imaging features of COVID-19.

\begin{tabular}{lll}
\hline Modality & GI & Pancreas \\
\hline Radiograph & $\begin{array}{l}\text { Portal venous gas suggesting bowel } \\
\text { infarction and perforation. }\end{array}$ & \\
USG & & $\begin{array}{l}\text { Voluminous pancreas without } \\
\text { gall stones. }\end{array}$ \\
& & \\
& & \\
CT & $\begin{array}{l}\text { Acute: Fluid-filled colon. } \\
\text { Progression: Bowel wall thickening, } \\
\text { pericolic fat stranding, pneumatosis } \\
\text { intestinalis, mural hyperenhancement. }\end{array}$ & $\begin{array}{l}\text { Acute: } \text { Pancreatic edema, } \\
\text { dilation of the pancreatic duct } \\
\text { with focal necrosis. } \\
\text { Dense opacification of the }\end{array}$ \\
& $\begin{array}{l}\text { Progression: } \\
\text { intestinal wall. }\end{array}$ & $\begin{array}{l}\text { Duodenal/periduodenal } \\
\text { inflammation. }\end{array}$
\end{tabular}

Liver Renal

$\mathrm{MRI}$

Increased liver fat $>10 \%$ in
severe disease.
Gall bladder distension, GB wall
thickening with GB
sludge-acute cholestasis.
Increased echogenicity of
hepatic parenchyma-fatty liver.
Heterogeneous liver with GB thickening.

Edema of the renal parenchyma Wedgeshaped low attenuation suggesting infarct.

\section{severe disease.}

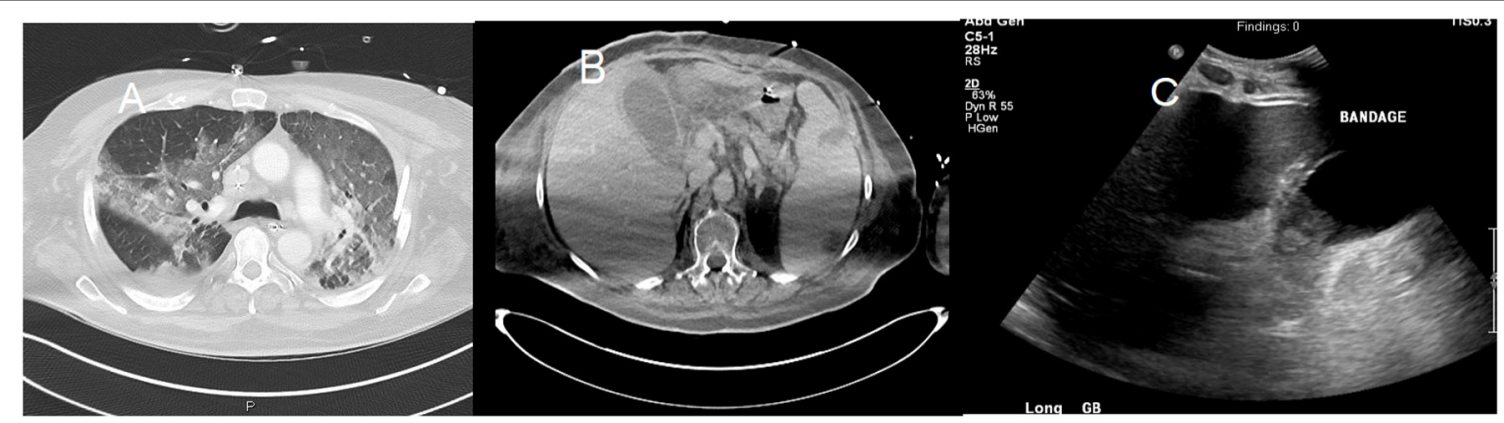

FIGURE 5 | A 58-year-old man with a past medical history of obesity, post-primary coronary intervention, and coronary artery disease, got admitted to the intensive care unit with severe hypoxia and tachycardia. The patient tested positive for COVID-19. (A) Chest CT showed patchy airspace opacities with predominant ground-glass opacities seen in the upper lobes and consolidative opacities with air bronchograms in the lower lobes. (B) Abdominal CT showed hepatosplenomegaly with splenic infarcts and perisplenic fluid collections. Gallbladder wall thickening with trace pericholecystic fluid was also noted, suggesting acalculous cholecystitis. (C) An abdominal ultrasound image of the same patient showed moderately distended and thickened walls of the gall bladder.

80, 81). Due to parenchymal inflammation, density around the intestinal wall mimicking a GGO specific to COVID-19 can be noted in CT (81). Abdominal CT has also been a remarkable diagnostic tool for COVID-19-associated cholecystitis, hepatitis, and splenic infarcts (Figure 5). Typical CT findings in patients with pancreatic complications varied from mild pancreatic edema and dilation of pancreatic duct to focal necrosis of pancreas with distinct inflammation involving second and third parts of the duodenum and periduodenal space, which was suggested to be specific to severe stages of COVID-19 (78, 79, 82). Moreover, there have been reports of renal involvements in abdominopelvic CT scans of COVID-19 patients, including parenchymal edema and more specifically wedge-shaped low attenuation region in the superior pole of the kidney, suggestive of renal infarct due to thromboembolism $(16,37)$.

\section{Magnetic Resonance Imaging}

Liver injury in COVID-19 patients is believed to be a consequence of either mechanical ventilation and/or severe inflammation and stress-induced injury (83). One potential method for the quantification of hepatitis severity is hepatic T1 mapping MRI, in which liver fat proportions of more than $10 \%$ have been shown to be associated with the severity of COVID-19 infection (84).

\section{DISCUSSION}

This multiorgan multimodality narrative review provided comprehensive information on the burgeoning evidence of varied clinical phenotypes of COVID-19. Studies have reported incidental COVID-19 findings in the lung bases observed in the non-pulmonary imaging of patients with non-COVID indications, including acute abdomen or preoperative scans $(85,86)$. This highlights how non-pulmonary imaging could be as important as pulmonary imaging in the diagnosis and management of patients, given the unraveled nature of COVID19. One of the major recommendations of ACR during this pandemic was the necessity for physicians to be able to recognize possible COVID-19-associated imaging findings even in patients with imaging not acquired for COVID-related complaints (30). 
As COVID-19 is a new disease, research relies on retrospective data collected from patients with both imaging and RT-PCR done and hence subjected to selection bias. When translating these reported findings to clinical practice, clinicians should exercise caution and pay attention to the characteristics of each study population, as population differences can result in conflicting results in diagnostic accuracy of the modalities (87). Additionally, the challenges of COVID-19 have also caused alterations and significant changes to the workflow of clinicians and radiologists as the health and safety of healthcare workers come into play along with patients. Diagnostic imaging services have been time-consuming and complicated by the need for strict infection control and prevention practices developed to contain the risk of transmission and protect healthcare personnel (88). Hence, the decision to image suspected patients or COVID-19-positive patients is based on their impact on the improvement of patient status. The ACR recommends not to use any advanced imaging as a screening test and only to use radiography and ultrasound as a first-line tool (11, 30). Even though radiographs are insensitive for the detection of specific signs of disease, equipment portability can favor this imaging modality in triaging and monitoring disease $(10,88)$. Ultrasound and echocardiography can provide useful insights that can be correlated with CT findings but need to be employed with certain caveats to ensure the safety of the healthcare provider. Especially in critically ill patients, the use of POCUS by the clinical provider for disease management can be helpful as formal echocardiograms can increase exposure to care providers and can be challenging to coordinate.

There are a few limitations to our review paper. Since this is a narrative review, we might have introduced selection bias in selecting the articles and literature in this review. Second, as the aim of this review was not to critically appraise literature, we did not assess the quality and validity of the literature and the evidence based on the predefined protocol. Third, we could not address the diagnostic value and specificity of imaging findings as, to our knowledge, only a few clinical studies were published comparing COVID and non-COVID imaging findings. Further comparative analysis is required. Lastly, at the time of the review

\section{REFERENCES}

1. Home. Johns Hopkins Coronavirus Resource Center [Internet]. (2020). Available online at: https://coronavirus.jhu.edu/ (accessed 2020 July 16).

2. Coronavirus Pandemic (COVID-19). The data-Our World in Data [Internet]. (2020). Available online at: https://ourworldindata.org/ coronavirus-data (accessed 2020 July 16).

3. Hamming I, Timens W, Bulthuis MLC, Lely AT, Navis GJ, van Goor H. Tissue distribution of ACE2 protein, the functional receptor for SARS coronavirus. A first step in understanding SARS pathogenesis. J Pathol. (2004) 203:631-7. doi: $10.1002 /$ path. 1570

4. Huang C, Wang Y, Li X, Ren L, Zhao J, Hu Y, et al. Clinical features of patients infected with 2019 novel coronavirus in Wuhan, China. Lancet. (2020) 395:497-506. doi: 10.1016/S0140-6736(20)30183-5

5. World Health Organization. Clinical management of severe acute respiratory infection (SARI) when COVID-19 disease is suspected. WHO/2019nCoV/clinical/20205 [Internet]. Available online at: https://www.who. was written, not much was established about the long-term effects of COVID-19, and hence, not much could be described as the role of imaging in long-term COVID-19.

Given that most clinical presentations of COVID19 have been non-specific and commonly observed in many other systemic diseases, this thorough review of reported imaging findings in this disease could be a potential guide for clinical practitioners, radiologists, and intensivists to diagnose early and improve the management of patients referred with these alarm signs. Since cycles of acceleration, suppression, and re-emergence of COVID19 have been predicted, multimodality imaging can play a crucial role in diagnosing and stratifying patients in the event of a second wave. Future research is needed to establish the long-term sequelae of COVID-19 infection on multiorgan function.

\section{CONCLUSION}

Imaging in complement with clinical examination and biomarkers can provide a detailed assessment and management of organ damage and dysfunction in COVID-19. In the era of COVID, all physicians should endeavor to carefully look for imaging features reported in COVID-19 patients, even in asymptomatic patients and in imaging other than chest radiographs, as patients with multisystem presentations have had unfavorable outcomes such as hospitalization, mechanical ventilation, and mortality.

\section{AUTHOR CONTRIBUTIONS}

VV: conceptualization, acquisition, and writing original draft. MS, BA, and JL: writing, reviewing, and editing. All authors contributed to the article and approved the submitted version.

\section{FUNDING}

The writing group has been funded by the National Heart and Lung Blood Institute (NHLBI), part of the National Institute of Health.

int/publications-detail-redirect/clinical-management- of-covid-19\#. XwBCr52OsJk.mendeley

6. Wu Z, McGoogan JM. Characteristics of and important lessons from the coronavirus disease 2019 (COVID-19) Outbreak in China: summary of a report of 72314 cases from the Chinese center for disease control and prevention. JAMA-J Am Med Assoc. (2020) 323:1239-42. doi: 10.1001/jama. 2020.2648

7. Wiersinga WJ, Rhodes A, Cheng AC, Peacock SJ, Prescott HC. Pathophysiology, transmission, diagnosis, and treatment of coronavirus disease 2019 (COVID-19): a review [Internet]. JAMA$J$ Am Med Assoc. (2020) 324:782-93. doi: 10.1001/jama.2020. 12839

8. Arentz M, Yim E, Klaff L, Lokhandwala S, Riedo FX, Chong $\mathrm{M}$, et al. Characteristics and Outcomes of 21 Critically Ill Patients with COVID-19 in Washington State. JAMA$J$ Am Med Assoc. (2020) 323:1612-4. doi: 10.1001/jama.2020. 4326 
9. Gupta A, Madhavan M V., Sehgal K, Nair N, Mahajan S, Sehrawat TS, et al. Extrapulmonary manifestations of COVID-19. Nat Med. (2020) 26:101732. doi: 10.1038/s41591-020-0968-3

10. Manna S, Wruble J, Maron SZ, Toussie D, Voutsinas N, Finkelstein $\mathrm{M}$, et al. COVID-19: A multimodality review of radiologic techniques, clinical utility, and imaging features. Radiol Cardiothorac Imaging. 2:e200210. doi: 10.1148/ryct.2020200210

11. Wong HYF, Lam HYS, Fong AHT, Leung ST, Chin TWY, Lo CSY, et al. Frequency and distribution of chest radiographic findings in COVID-19 positive patients. Radiology. (2019) 27:201160. doi: 10.1148/radiol.2020201160

12. Rudski L, Januzzi JL, Rigolin VH, Bohula EA, Blankstein R, Patel $\mathrm{AR}$, et al. Multimodality imaging in evaluation of cardiovascular complications in patients with COVID-19. J Am Coll Cardiol. (2020) 6:80 doi: 10.1016/j.jacc.2020.06.080

13. Malik P, Patel U, Mehta D, Patel N, Kelkar R, Akrmah M, et al. Biomarkers and outcomes of COVID-19 hospitalisations: systematic review and meta-analysis. BMJ Evidence-Based Med. 26:107-8. doi: 10.1136/bmjebm-2020-111536

14. Zu ZY, Jiang M Di, Xu PP, Chen W, Ni QQ, Lu GM, et al. Coronavirus Disease 2019 (COVID-19): a perspective from China. Radiology. (2020) 21:200490. doi: 10.1148/radiol.2020200490

15. Ng M-Y, Lee EY, Yang J, Yang F, Li X, Wang H, et al. Imaging profile of the COVID-19 infection: Radiologic findings and literature review. Radiol Cardiothorac Imaging. 2:e200034. doi: 10.1148/ryct.2020200034

16. Lushina N, Kuo JS, Shaikh HA. Pulmonary, cerebral, and renal thromboembolic disease associated with COVID-19 infection. Radiology. 20:1623. doi: 10.1148/radiol.2020201623

17. Vetrugno L, Bove T, Orso D, Barbariol F, Bassi F, Boero E, et al. Our Italian experience using lung ultrasound for identification, grading and serial followup of severity of lung involvement for management of patients with COVID19 [Internet]. London: Blackwell Publishing Inc. (2020). Available online at: https://www.ncbi.nlm.nih.gov/pmc/articles/PMC7228311/ (accessed July 3, 2020).

18. Testa A, Soldati G, Copetti R, Giannuzzi R, Portale G, Gentiloni-Silveri N. Early recognition of the 2009 pandemic influenza A (H1N1) pneumonia by chest ultrasound. Crit Care. (2012) 16:202. doi: 10.1186/cc11201

19. Xing C, Li Q, Du H, Kang W, Lian J, Yuan L. Lung ultrasound findings in patients with COVID-19 pneumonia. Crit Care. (2020) 24:174. doi: 10.1186/s13054-020-02876-9

20. Johri AM, Galen B, Kirkpatrick JN, Lanspa M, Mulvagh S, Thamman R. ASE statement on point-of-care ultrasound during the 2019 novel coronavirus pandemic. J Am Soc Echocardiogr. 33:670-3. doi: 10.1016/j.echo.2020.04.017

21. Buonsenso D, Pata D, Chiaretti A. COVID-19 outbreak: less stethoscope, more ultrasound. Lancet. (2020) 8:e27. doi: 10.1016/S2213-2600(20)30120-X

22. Ai T, Yang Z, Hou H, Zhan C, Chen C, Lv W, et al. Correlation of Chest CT and RT-PCR testing in coronavirus disease 2019 (COVID-19) in China: a report of 1014 Cases. Radiology. 26:200642. doi: 10.1148/radiol.2020200642

23. Bao C, Liu X, Zhang H, Li Y, Liu J. Coronavirus Disease 2019 (COVID-19) CT findings: a systematic review and meta-analysis. J Am Coll Radiol. (2020) 17:3052. doi: 10.1016/j.jacr.2020.03.006

24. Sun Z, Zhang N, Li Y, Xu X. A systematic review of chest imaging findings in COVID-19. Quant Imaging Med Surg. (2020) 10:105879. doi: 10.21037/qims-20-564

25. Carotti M, Salaffi F, Sarzi-Puttini P, Agostini A, Borgheresi A, Minorati D, et al. Chest CT features of coronavirus disease 2019 (COVID-19) pneumonia: key points for radiologists [Internet]. Radiologia Medica. (2020) 125:63646. doi: 10.1007/s11547-020-01237-4

26. Yang W, Sirajuddin A, Zhang X, Liu G, Teng Z, Zhao S, et al. The role of imaging in 2019 novel coronavirus pneumonia (COVID-19). Eur Radiol. (2020) 4:1. doi: 10.1007/s00330-020-06827-4

27. Ye Z, Zhang Y, Wang Y, Huang Z, Song B. Chest CT manifestations of new coronavirus disease 2019 (COVID-19): a pictorial review. Eur Radiol [Internet]. (2020) 23:1. doi: 10.1007/s00330-02006801-0

28. Kumar Venugopal V, Mahajan V, Rajan S, Agarwal V, Rajan R, Syed S, et al. A systematic meta-analysis of ct features of COVID-19: Lessons from Radiology. (2020). Available online at: https://doi.org/10.1101/2020.04.04. 20052241 (accessed July 2, 2020) doi: 10.1101/2020.04.04.20052241
29. Altmayer S, Zanon M, Pacini GS, Watte G, Barros MC, Mohammed T-L, et al. Comparison of the computed tomography findings in COVID-19 and other viral pneumonia in immunocompetent adults: a systematic review and meta-analysis. Eur Radiol. (2020) 30:1. doi: 10.1007/s00330-020-07018-x

30. ACR. Recommendations for the use of Chest Radiography and Computed Tomography (CT) for Suspected COVID-19 Infection | American College of Radiology [Internet]. Available online at: https://www.acr.org/Advocacyand-Economics/ACR-Position-Statements/Recommendations-for-ChestRadiography-and-CT-for-Suspected-COVID19-Infection (accessed 2020 July 16).

31. Vasilev Y, Sergunova K, Bazhin A, Masri A, Vasileva Y, Suleumanov E, et al. MRI of the lungs in patients with COVID-19: clinical case. medRxiv. (2020) 88:372

32. Zou S, Zhu X. FDG PET/CT of COVID-19. Radiology. 2020:200770. doi: 10.1148/radiol.2020200770

33. Treglia G. The role of 18F-FDG PET for COVID-19 infection: myth versus reality [Internet]. Clinical and Translational Imaging. New York, NY: Springer (2020). Available online at: https://www.ncbi.nlm.nih.gov/pmc/articles/ PMC7191553/ (accessed July 1, 2020). doi: 10.1007/s40336-020-00367-z

34. Guo $\mathrm{T}$, Fan $\mathrm{Y}$, Chen $\mathrm{M}$, Wu $\mathrm{X}$, Zhang $\mathrm{L}, \mathrm{He} \mathrm{T}$, et al. Cardiovascular implications of fatal outcomes of patients with coronavirus disease 2019 (COVID-19). JAMA Cardiol. (2020) 5:811-8. doi: 10.1001/jamacardio.2020.1017

35. Madjid M, Safavi-Naeini P, Solomon SD, Vardeny O. Potential effects of coronaviruses on the cardiovascular system: a review. JAMA Cardiol. (2020) 10:1-10. doi: 10.1001/jamacardio.2020.1286

36. Inciardi RM, Lupi L, Zaccone G, Italia L, Raffo M, Tomasoni D, et al. Cardiac Involvement in a patient with coronavirus disease 2019 (COVID-19). JAMA Cardiol. (2020) 2019:1-6. doi: 10.1001/jamacardio.2020.1096

37. Li Z, Wu M, Yao J, Guo J, Liao X, Song $\mathrm{S}$, et al. Caution on Kidney Dysfunctions of COVID-19 Patients. SSRN Electron J. (2020) 20:125. doi: 10.2139/ssrn.3559601

38. Bonow RO, Fonarow GC, O'Gara PT, Yancy CW. Association of Coronavirus Disease 2019 (COVID-19) with myocardial injury and mortality. JAMA Cardiol. (2020) 22:19362 doi: 10.1001/jamacardio.2020.1105

39. Peng QY, Wang XT, Zhang LN. Using echocardiography to guide the treatment of novel coronavirus pneumonia [Internet]. Critical Care. (2020) 24:143. doi: 10.1186/s13054-020-02856-Z

40. Mahmoud-Elsayed HM, Moody WE, Bradlow WM, Khan-Kheil AM, Hudsmith LE, Steeds RP. Echocardiographic Findings in Covid-19 Pneumonia. Can J Cardiol. (2020) 28:107-8. doi: 10.1016/j.cjca.2020.05.030

41. Minhas AS, Scheel P, Garibaldi B, Liu G, Horton M, Jennings M, et al. Takotsubo Syndrome in the Setting of COVID-19. JACC Case Reports. (2020). Available online at: https://doi.org/10.1016/j.jaccas.2020.04.023 (accessed July 3, 2020). doi: 10.1016/j.jaccas.2020.04.023

42. Nguyen D, Nguyen T, De Bels D, Castro Rodriguez J. A case of Takotsubo cardiomyopathy with COVID 19. Eur Hear J-Cardiovasc Imaging. (2020) 21:1052. doi: 10.1093/ehjci/jeaa152

43. Ming Wang TK, Tang WHW, Flamm SD, Griffin B, Dugar S, Grimm RA, et al. The role of cardiac imaging in hospitalized COVID-19-positive patients: posted may 1, 2020. Cleve Clin J Med. 87:1-4. doi: 10.3949/ccjm.87a.ccc020

44. Churchill TW, Bertrand PB, Bernard S, Namasivayam M, Churchill J, Crousillat D, et al. Echocardiographic Features of COVID-19 Illness and Association with Cardiac Biomarkers. J Am Soc Echocardiogr. (2020) 5:28. doi: 10.1016/j.echo.2020.05.028

45. Argulian E, Sud K, Vogel B, Bohra C, Garg VP, Talebi S, et al. Right ventricular dilation in hospitalized patients with COVID-19 Infection. JACC Cardiovasc Imaging. (2020) 5:10. doi: 10.1016/j.jcmg.2020.05.010

46. Lan Y, Liu W, Zhou Y. Right Ventricular Damage in COVID-19: Association Between Myocardial Injury and COVID-19. Front Cardiovasc Med. (2021) 0:82. doi: $10.3389 /$ fcvm.2021.606318

47. P K, LB C, R R, M A, W Z, G G, et al. Biventricular strain by speckle tracking echocardiography in COVID-19: findings and possible prognostic implications. Future Cardiol. 17:663-7. doi: 10.2217/fca-2020-0100

48. X L, X M. Acute respiratory failure in COVID-19: is it "typical" ARDS? Crit Care. (2020) 24:845. doi: 10.1186/s13054-020-02911-9

49. Poissy J, Goutay J, Caplan M, Parmentier E, Duburcq T, Lassalle F, et al. Pulmonary embolism in COVID-19 patients: 
awareness of an increased prevalence. Circulation. (2020) 24:2. doi: 10.1161/CIRCULATIONAHA.120.047430

50. Fauvel C, Weizman O, Trimaille A, Mika D, Pommier T, Pace $\mathrm{N}$, et al. Pulmonary embolism in COVID-19 patients: a French multicentre cohort study Pulmonary circulation. Eur Heart J. (2020) 500:1-11. doi: 10.1093/eurheartj/ehaa500

51. Bikdeli B, Madhavan M V., Jimenez D, Chuich T, Dreyfus I, Driggin E, et al. COVID-19 and Thrombotic or thromboembolic disease: implications for prevention, antithrombotic therapy, and follow-up: JACC state-of-the-art review. J Am Coll Cardiol. (2020) 32:2950-73. doi: 10.1016/j.jacc.2020.04.031

52. Szekely Y, Lichter Y, Taieb P, Banai A, Hochstadt A, Merdler I, et al. The spectrum of cardiac manifestations in coronavirus disease 2019 (COVID-19). Systematic Echocardiographic Study. (2020) 20:1. doi: 10.1161/CIRCULATIONAHA.120.047971

53. Jain SS, Liu Q, Raikhelkar J, Fried J, Elias P, Poterucha TJ, et al. Indications and findings on transthoracic echocardiogram in COVID-19. J Am Soc Echocardiogr. (2020) 33:1278-1284. doi: 10.1016/j.echo.2020.06.009

54. Kirkpatrick JN, Mitchell C, Taub C, Kort S, Hung J, Swaminathan M. ASE statement on protection of patients and echocardiography service providers during the 2019 novel coronavirus outbreak: endorsed by the american college of cardiology. J Am Soc Echocardiogr. (2020) 33:64853. doi: 10.1016/j.echo.2020.04.001

55. Luetkens JA, Isaak A, Zimmer S, Nattermann J, Sprinkart AM, Boesecke C, et al. Diffuse Myocardial Inflammation in COVID-19 Associated Myocarditis Detected by Multiparametric Cardiac Magnetic Resonance Imaging [Internet]. Circulation. 30:10897. doi: 10.1161/CIRCIMAGING.120.010897

56. B K A, Chaudhuri D. A review of acute myocardial injury in coronavirus disease 2019. Cureus. (2020) 12:226.. doi: 10.7759/cureus. 8426

57. Han Y, Chen T, Bryant J, Bucciarelli-Ducci C, Dyke C, Elliott MD, et al. Society for Cardiovascular Magnetic Resonance (SCMR) guidance for the practice of cardiovascular magnetic resonance during the COVID-19 pandemic. $J$ Cardiovasc Magn Reson. (2020) 22:26. doi: 10.1186/s12968-020-00628-w

58. Oudkerk M, Büller HR, Kuijpers D, van Es N, Oudkerk SF, McLoud TC, et al. Diagnosis, Prevention, and Treatment of Thromboembolic Complications in COVID-19: report of the national institute for public health of the Netherlands. Radiology. (2020) 20:1629. doi: 10.1148/radiol.2020201629

59. Wu X, Wang C, Zhang C, Zhang Y, Ding F, Yan J. Computed tomography for detecting left atrial thrombus: a meta-analysis. Archiv Med Sci. (2012) 16:943-51. doi: 10.5114/aoms.2012.32400

60. Society of Cardiology E. ESC Guidance for the Diagnosis and Management of CV Disease during the COVID-19 Pandemic. (2020).

61. Siripanthong B, Nazarian S, Muser D, Deo R, Santangeli P, Khanji MY, et al. Recognizing COVID-19-related myocarditis: the possible pathophysiology and proposed guideline for diagnosis and management. Hear Rhythm. (2020) 32:387246. doi: 10.1016/j.hrthm.2020.05.001

62. Kim I-C, Kim JY, Kim HA, Han S. COVID-19-related myocarditis in a 21-year-old female patient. Eur Heart J. (2020) 41:1859. doi: 10.1093/eurheartj/ehaa288

63. Fotuhi M, Mian A, Meysami S, Raji CA. Neurobiology of COVID-19. J Alzheimer's Dis. (2020) 76:3-19. doi: 10.3233/JAD-200581

64. Qureshi AI, Abd-Allah F, Alsenani F, Aytac E, Borhani-Haghighi A, Ciccone A, et al. Management of acute ischemic stroke in patients with COVID-19 infection: Report of an international panel [Internet]. Int J Stroke. (2020) 12:344. doi: $10.1177 / 1747493020923234$

65. Khosravani H, Rajendram P, Notario L, Chapman MG, Menon BK. Protected Code Stroke: Hyperacute Stroke Management during the Coronavirus Disease 2019 (COVID-19) Pandemic [Internet]. Stroke. (2019) 51:18915. doi: 10.1161/STROKEAHA.120.029838

66. Baracchini C, Pieroni A, Viaro F, Cianci V, Cattelan AM, Tiberio I, et al. Acute stroke management pathway during Coronavirus-19 pandemic. Neurol Sci. (2020) 41:1003-5. doi: 10.1007/s10072-020-04375-9

67. Mahammedi A, Saba L, Vagal A, Leali M, Rossi A, Gaskill M, et al. Imaging in Neurological Disease of Hospitalized COVID-19 Patients: an Italian multicenter retrospective observational study. Radiology. (2019) 2019:33. doi: 10.1148/radiol.2020201933

68. Qureshi AI, Baskett WI, Huang W, Shyu D, Myers D, Raju M, et al. Acute ischemic stroke and COVID-19. Stroke. (2021) 15:90512. doi: 10.1161/STROKEAHA. 120.031786
69. Neurology. COVID-19 Protocols [Internet]. (2020). Available online at: https:// covidprotocols.org/protocols/neurology/ (accessed July 23, 2020).

70. Toscano G, Palmerini F, Ravaglia S, Ruiz L, Invernizzi P, Cuzzoni MG, et al. Guillain-Barré Syndrome Associated with SARS-CoV-2. N Engl J Med. (2020) 9:91. doi: 10.1056/NEJMc2009191

71. Politi LS, Salsano E, Grimaldi M. Magnetic Resonance imaging alteration of the brain in a patient with coronavirus disease 2019 (COVID-19) and Anosmia [Internet]. JAMA Neurol. (2020) 77:1028-29. doi: 10.1001/jamaneurol.2020.2125

72. Avula A, Nalleballe K, Narula N, Sapozhnikov S, Dandu V, Toom S, et al. COVID-19 presenting as stroke. Brain Behav Immun. (2020) 87:115. doi: 10.1016/j.bbi.2020.04.077

73. Radmanesh A, Derman A, Lui YW, Raz E, Loh JP, Hagiwara M, et al. COVID19 -associated Diffuse Leukoencephalopathy and Microhemorrhages. Radiology. (2020) 21:202040. doi: 10.1148/radiol.2020202040

74. Poyiadji N, Shahin G, Noujaim D, Stone M, Patel S, Griffith B. COVID-19-associated acute hemorrhagic necrotizing encephalopathy: CT and MRI features. Radiology. (2020) 31:201187. doi: 10.1148/radiol.20202 01187

75. Kremer S, Lersy F, de Sèze J, Ferré J-C, Maamar A, Carsin-Nicol B, et al. Brain MRI findings in severe COVID-19: a retrospective observational study. Radiology. (2020) 16:202222. doi: 10.1148/radiol.2020 202222

76. Pan L, Mu M, Yang P, Sun Y, Wang R, Yan J, et al. Clinical characteristics of COVID-19 patients with digestive symptoms in Hubei, China: A descriptive, cross-sectional, multicenter study. Am J Gastroenterol. (2020) 115:76673. doi: 10.14309 /ajg.0000000000000620

77. Bhayana R, Som A, Li MD, Carey DE, Anderson MA, Blake MA, et al. abdominal imaging findings in COVID-19: preliminary observations. Radiology. (2020) 20:1908. doi: 10.1148/radiol.2020201908

78. Szatmary P, Arora A, Raraty MGT, Dunne DFJ, Baron RD, Halloran CM. Emerging phenotype of SARS-CoV2 associated pancreatitis. Gastroenterology. (2020) 5:69. doi: 10.1053/j.gastro.2020. 05.069

79. Hadi A, Werge M, Kristiansen KT, Pedersen UG, Karstensen JG, Novovic S, et al. Coronavirus Disease-19 (COVID-19) associated with severe acute pancreatitis: case report on three family members. Pancreatology. (2020) 20:665-7. doi: 10.1016/j.pan.2020. 04.021

80. Carvalho A, Alqusairi R, Adams A, Paul M, Kothari N, Peters S, et al. SARS-CoV-2 gastrointestinal infection causing hemorrhagic colitis: implications for detection and transmission of COVID-19 disease. Am J Gastroenterol. (2020) 115:942-6. doi: 10.14309/ajg.0000000000 000667

81. Takifuji K. Computerized tomography scan findings of a patient with severe enterocolitis associated with the coronavirus disease 2019: a case report. (2019), 1-12. doi: 10.21203/rs.3.rs-21006/v1

82. Liu F, Long X, Zou W, Fang M, Wu W, Li W, et al. Highly ACE2 Expression in Pancreas May Cause Pancreas Damage After SARS-CoV-2 Infection. Available online at: https:// doi.org/10.1101/2020.02.28.20029181 doi: $\quad 10.1101 / 2020.02 .28$. 20029181

83. Zahedi M, Yousefi M, Abounoori M, Malekan M, Tajik F, Heydari $\mathrm{K}$, et al. Liver Function in Novel Coronavirus Disease (COVID19): A Systematic Review and Meta-Analysis. Available online at: https://doi.org/10.1101/2020.05.20.20108357 doi: 10.1101/2020.05.20. 20108357

84. Banerjee R. High Liver Fat Associates With Higher Risk Of Developing Symptomatic Covid-19 Infection-Initial Uk Biobank Observations. Available online at: https://doi.org/10.1101/2020. 06.04.20122457 (accessed 2020 July 4). doi: 10.1101/2020.06.04. 20122457

85. Hamilton NE, Adam GH, Ifan DL, Lam SS, Johnson K, Vedwan KAG, et al. Diagnostic utility of additional whole-chest CT as part of an acute abdominal pain CT imaging pathway during the COVID-19 pandemic. Clin Radiol. (2020) 75:345. doi: 10.1016/j.crad.2020.06.002

86. Siegel A, Chang PJ, Jarou ZJ, Paushter DM, Harmath CB, Arevalo J Ben, et al. Lung base findings of coronavirus disease (COVID-19) on abdominal 
ct in patients with predominant gastrointestinal symptoms. Am J Roentgenol. (2020) 20:23232. doi: 10.2214/AJR.20.23232

87. Eng J, Bluemke DA. Imaging publications in the COVID-19 pandemic: applying new research results to clinical practice. Radiology. (2020) 23:201724. doi: 10.1148/radiol.2020201724

88. Rubin GD, Ryerson CJ, Haramati LB, Sverzellati N, Kanne JP, Raoof $\mathrm{S}$, et al. The Role of Chest Imaging in patient management during the COVID-19 pandemic: a multinational consensus statement from the fleischner society. Radiology. (2020) 296:172-80. doi: 10.1148/radiol.2020 201365

Conflict of Interest: The authors declare that the research was conducted in the absence of any commercial or financial relationships that could be construed as a potential conflict of interest.
Publisher's Note: All claims expressed in this article are solely those of the authors and do not necessarily represent those of their affiliated organizations, or those of the publisher, the editors and the reviewers. Any product that may be evaluated in this article, or claim that may be made by its manufacturer, is not guaranteed or endorsed by the publisher.

Copyright $\odot 2021$ Varadarajan, Shabani, Ambale Venkatesh and Lima. This is an open-access article distributed under the terms of the Creative Commons Attribution License (CC BY). The use, distribution or reproduction in other forums is permitted, provided the original author(s) and the copyright owner(s) are credited and that the original publication in this journal is cited, in accordance with accepted academic practice. No use, distribution or reproduction is permitted which does not comply with these terms. 